\title{
Tamanho de parcela amostral para inventários florestais (1).
}

\author{
Niro Higuchi ${ }^{2}$ ) \\ Joaquim dos Santos $\left({ }^{2}\right)$ \\ Fernando C. S. Jardim ( $\left.{ }^{2}\right)$
}

\begin{abstract}
Resumo
Resultado da investigaçāo sobre o tamanho ideal de parcela amostral para Inventários Florestais, feita com base em 32 diferentes tamanhos, simulados, um a um, sobre o Inventário Florestal a $100 \%$ de uma área de 80 hectares de floresta tropical úmida de terra firme, com árvores de DAP maior ou igual a $25 \mathrm{~cm}$. A parcela que apresentou os melhores resultados foi a de dimensōes de $37,5 \times 150$ metros.
\end{abstract}

\section{INTRODUÇÃo}

Atualmente, com a crescente valorização da terra e de sua cobertura vegetal, mais se tornam necessários os Inventários Florestais, os quais permitem descrever a quantidade e a qualidade das espécies florestais ocorrentes e muitas das características do terreno. A cada dia que passa, o conceito de Inventário Florestal se torna mais abrangente, de acordo com a utilização de áreas florestais para valores não madeireiros, tais como a recreação, manejo de bacias hidrográficas, fontes de energia, vida silvestre e possível conversão para outros usos da terra. Normalmente, o Inventário Florestal é o primeiro passo para lançar a base de pesquisas referentes a recursos naturais e também para tomada de qualquer decisão relacionada ao uso da teria.

$\mathrm{Na}$ região Amazônica, mais do que em qualquer outra região do mundo, é praticamente impossivel fazer uma completa enumeração dos individuos de uma população florestal, porque $o$ custo e o tempo necessários para tal fim seriam imensuráveis. Uma maneira mais fácil e eficiente para fazer a avaliação do potencial florestal de uma região é através de amostragem, a qual tem condições de oferecer a necessária informação a um menor custo e maiş rapidamente. Segundo Husch et al. (1971) uma outra vantagem, freqüentemente não reconhecida, é que um procedimento de amostragem pode produzir resultados mais confiáveis que uma completa enumeração. Esta afirmativa se deve ao fato que, medindo somente uma porção da floresta, maior cuidado pode ser dispensado em menos medições, a supervisão é mais fácil, menor e melhor treinado o número de pessoas no trabalho de campo e a provável redução de erros năo amostrais.

A teoria de amostragem aplicada em Inventário Florestal data do século XIX na Europa. No Brasil, os primeiros trabalhos de Inventário Florestal pubiicados, iniciaram com os primeiros convênios do Governo brasileiro com a FAO (Food and Agriculture Organization) na década de 50 , ganhando mais impulso a partir da criação do primeiro curso de Engenharia Florestal no Brasil, no início dos anos 60.

É fundamental compreender que não existe, entretanto, um sistema de amostragem que pode ser universalmente aplicado. Para cada situação florestal existe um adequado tipo de amostragem. O método a usar é o produto final de uma série de considerações, cada uma tendo uma influência sobre como a amostragem será levado a cabo.

Segundo Husch et al. (1971) e Loetsch et al. (1973), o método de amostragem para encontrar os objetivos do Inventário Florestal é determinado pelo tipo de unidade de amostragem, seu tamanho e forma, o número a ser empregado e a maneira de seleção e a distribuição sobre a área florestal, seguidos pelos procedimentos para medições nas unidades selecionadas e análise dos dados resultantes.

(1) - Projeto "Manejo Ecológico e Exploraçāo da Floresta Tropical Úmida". Convênio INPA/BID/FINEP.

(2) - Instituto Nacional de Pesquisas da Amazônia, Manaus. 
Alguns trabaihos de Inventário Florestal exemplificam a utilização de diferentes tipos de amostragem no Brasil (Tabela 1) .

Da Tabela anterior, nota-se que mesmo em diferentes regiões, o tipo de amostragem pode ser repetido, desde que haja clara caracterização cios tipos florestais, independentemente de localização geográfica. Entretanto, os tamanhos das parcelas utilizadas nesses trabaIhos de Inventário Florestal variam substancialmente, mesmo quando se utiliza o mesmo tipo de amostragem e executado por um mesmo grupo de trabalho como são os 5 primeiros trabaihos da Tabela, os quais foram executados por pesquisadores do Curso de Engenharia Florestal da Universidade Federal do Paraná. $\mathrm{Em}$ todos os trabalhos, existem explicações para os diferentes tipos de amostragem mas não para os diferentes tamanhos de parcelas.

Em outros países, mesmo sendo desenvolvidos e com grande tradição florestal, a coincidência de tamanho de parcelas amostrais é rara, como é mostrada na Tabela 2.

Para o Inventário Florestal Nacional de Surinamee, segundo De Milde (1975), foi utilizado a amostragem em 3 estágios com parcelas circulares de 0,04 ha.

Eni Fiji, Berry \& Howard (1973), numa região tropical, utilizaram a amostragem Siste- mática para o Inventário Florestal Nacional com parcelas de $20 \times 50 \mathrm{~m}$, sem justificar a razão do uso desse tamanho de parcela amostral.

Queiroz (1977) concluiu que o tamanho ideal para a subunidade do conglomerado nas florestas do Planalto do Tapajós é de 20×160m.

Segundo Husch et al. (1971), as estimativas não tendenciosas de volume, área basal e de outros parâmetros da população florestal, podem ser obtidas de qualquer tamanho ou forma das parcelas, variando significativamente, entretanto, a precisão e o custo do Inventário Florestal. E mais, quanto maior o tamanho da parcela para uma dada intensidade de amostragem, menor será o número de parcelas e menor será o tempo de viagem (deslocamento da equipe de campo) e estabelecimento das parcelas. Entretanto, o tempo de medição por parcela será maior. Onde o acesso é difícil, a questão de tempo de locomcção até a parcela eleita assume importância fundamental e, neste caso, quanto mais concentradas as parcelas, melhor e menos caro será o custo de viagem.

Em tlorestas heterogêneas, normalmente em regiões tropicais, as unidades amostrais pequenas podern resultar num número maior de unidades que contém árvores não mensuráveis, iornando a aplicação da teoria de distri-

TABELA 1 - Inventários Florestais no Brasil

\begin{tabular}{l|l|l}
\hline Local & Amostragem & Parcela \\
\hline Distrito Federal & Estratificada & $10 \times 500 \mathrm{~m}$ \\
PIC Altamira & Conglomerado & $10 \times 250 \mathrm{~m}$ \\
Sul do Brasil (Pinheiro) & Estratificada & $20 \times 125 \mathrm{~m}$ \\
Itaipu & Estratificada & Circular $1000 \mathrm{~m}^{2}$ \\
Centro-Oeste (Reflorest.) & Estratificada & $20 \times 30 \mathrm{~m}$ \\
JARI (Nativas) & Sistemática & $10 \times 200 \mathrm{~m}$ \\
JARI (Plantaçōes) & Int. Aleatória & Circular $500 \mathrm{~m}^{2}$ \\
Pará e Maranhäo & Sistemática & $10 \times 1000 \mathrm{~m}$ \\
Curuá-Una & Sistemática & $100 \times 40 \mathrm{~m}$ \\
Tapajós/Xíngu & Sistemática & $10 \times 1000 \mathrm{~m}$ \\
\hline
\end{tabular}

FONTE: UFPr. C.E.F. - 1972, 1976, 1978a. 1978b, 1980 e Heinsdijk, 1965; Glerum, 1965a, 1965 b. 
TABELA 2 - Inventários florestais em outros paises

\begin{tabular}{l|l|l}
\hline Pais & Tipo de Inv. Florestal & Parcela (ha) \\
\hline Inglaterra & Levantamento Nacional & 0,04 \\
Est. Unidos & Madeira de lei p/ serraria & 0,08 \\
& Vareta de madeira de lei & 0,04 \\
& Mudas & 0,004 \\
Suécia & Levantamento Nacional & 0,0138 \\
Finlândia & Levantamento Nacional & 0,1 \\
Japắo & Madeira de lei & 0,05 a 0,2 \\
Canadá & Madeira de lei & 0,08 a 0,1 \\
Alemanha & Madeira de lei & 0,01 a 0,05 \\
\hline
\end{tabular}

FONTE : Husch et. al. (1971).

buiçãs normal, inapropriada (Husch et al., 1971). Nesses casos, as maiores parcelas tem-se comprovado mais úteis dada a maior heterogeneidade. O princípio na escolha do tamanho e forma da parcela deveria ser de maneira a ter uma parcela bastante grande para incluir um número representativo de árvores mas, bastante pequena para garantir o tempo mínimo de mediçöes.

Spurr (1952), citado por Husch et al. (1971), recomenda que o tamanho da parcela deveria ser suficientemente grande para incluir, pelo menos, 20 árvores de tamanho mensurável. Essa afirmativa, entretanto, é bastante subjetiva porque não é especificado a dimensão exata para considerar uma árvore como mensurável.

Estabelecido o tamanho de uma parcela que pode ser um elemento ou um indivíduo (como uma árvore) ou unidade de medida (como 1 hectare), o tamanho de uma determinada população e de quaisquer de suas sub-divisões, devem ser mensuráveis (FAO, 1974).

Naturalmente, o objetivo de qualquer Inventário Florestal é obter o máximo de informações de uma floresta com a máxima precisão e um mínimo custo. Esses 3 fatores desempenham papel preponderante no planejamento de um Inventário Florestal e estão diretamente ligados ao tamanho da parcela e, conseqüentemente, à intensidade de amostra. gem.
Freese (1967) sugere que o objetivo do planejador de um levantamento florestal deveria ser de tomar observações suficientes para obter a precisão desejadi - nem mais e nem menos. E ainda, o número de observações necessárias depencie da precisão desejada e da variabilidade inerente da população que está sendo amostrada.

Por essas razões e pelo fato de não haver uma definição exata quanto ao tamanho de parcela amostral para Inventário Florestal, principalmente na região amazônica, o presente trabalho pretende, após várias simulações em dados de um Inventário Florestal a $100 \%$ de uma área de 80 hectares, apresentar o tamanho ideal de parcelas para futuros trabalhos de Inventário Florestal, se não para toda Amazônia, peio menos para o Distrito Agropecuário da SUFRAMA (Superintendência da Zona Franca de Manaus). Neste trabalho, a ênfase é dada para a precisão do Inventário, em relação aos diferentes tamanhos de parcelas amostrais e ao tempo de medição.

\section{MATERIAL E MÉTODOS}

A simulação de diferentes tamanhos e, naturalmente, formas de parcelas amostrais foi feita sobre uma rede de dados de um Inventário Florestal a $100 \%$. Este Inventário foi realizado na altura do $\mathrm{km}-23$ da vicinal ZF-2 do Distrito Agropecuário da SUFRAMA, área esta destinada a estudos de regeneração natural do projeto "Manejo Ecológico e Exploração da Floresta Tropical Úmida", aproximadamente a $90 \mathrm{~km}$ de Manaus, saindo pela Rodovia ManausCaracaraí.

A área inventariada constituiu-se de 3 blocos com 6 sub-blocos cada um (tratamentos de exploração florestal). Cada bloco, repetição do experimento, foi marcado com 24 hectares, sendo que no primeiro foi preciso deslocar os sub-blocos 1 e 3 para fora de suas posições originais dada a mudança do traçado da estrada de escoamento de madeira. Entretanto, para efeito de simulações, estes 2 subblocos foram incluídos na pesquisa, perfazendo assim um total de 80 hectares inventariados a $100 \%$. 
BLOCO

\begin{tabular}{|c|c|c|}
\hline $\mathrm{SB}_{1}$ & $\mathrm{SB}_{3}$ & $\mathrm{SB}_{5}$ \\
\hline $\mathrm{SB}_{2}$ & $\mathrm{SB}_{4}$ & $\mathrm{SB}_{6}$ \\
\hline
\end{tabular}

$$
\begin{aligned}
& \mathrm{SB}_{1}, \ldots \mathrm{SB}_{6}: \text { Sub-blocos } \\
& \mathrm{P} 1, \mathrm{P} 2 \ldots \mathrm{P} 8 \text { : Picadas } \\
& \mathrm{SP}_{1}, \ldots \mathrm{SP}_{8}: \text { Sub-parcelas }
\end{aligned}
$$

SUB-BLOCO

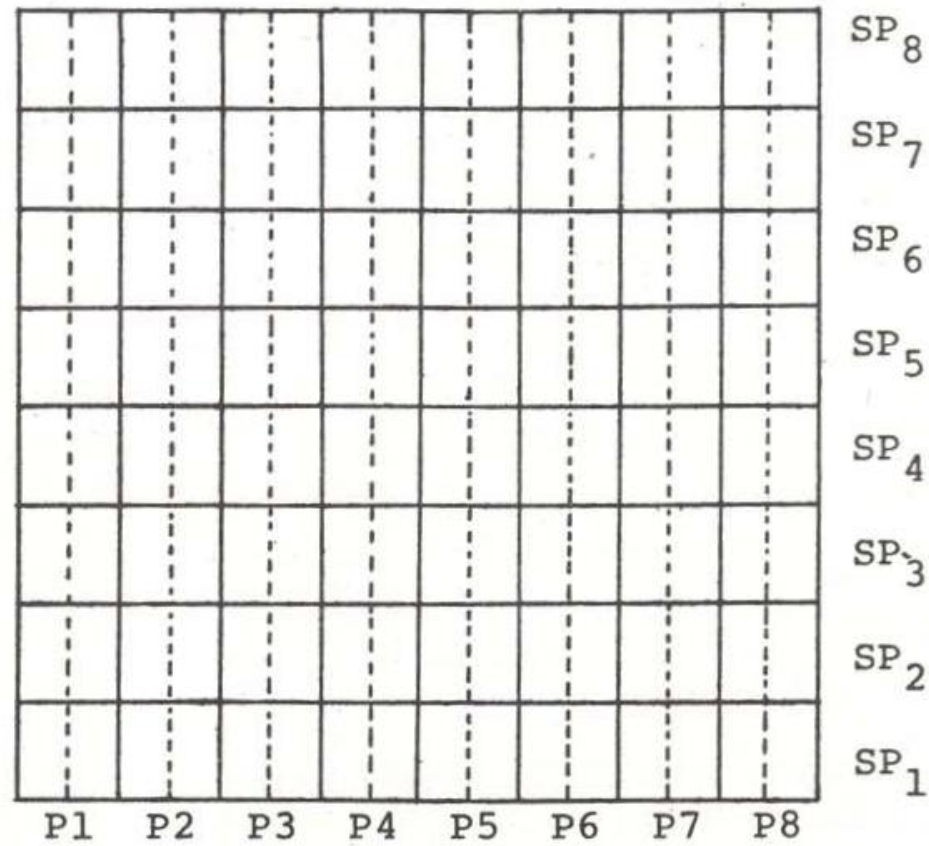

$200 \mathrm{~m}$
$P_{8}$

$\mathrm{SP}_{7}$

${ }^{5} 6$

$\mathrm{SP}_{5}$

⿷

응

$\mathrm{SP}_{3}$

$\mathrm{SP}_{2}$

$\mathrm{SP}_{1}$
Cada sub-bloco foi dividido por 8 picadas distando $25 \mathrm{~m}$ uma da outra. A linha da picada foi ainda subdividida em subparcelas de $25 \times 25 \mathrm{~m}$ para facilitar as medições e evitar os erros não amostrais. O Inventário Florestal foi feito ao longo das picadas, sendo diferenciado o lado direito do lado esquerdo, do centro da picada.

Dentro de cada subparcela foram coletados dados de todas as árvores com diâmetro à altura do peito (DAP) maior ou igual a $25 \mathrm{~cm}$ e, antados na ficha de campo, o nome da espécie florestal e sua classificação quanto à qualidade do fuste. Todas as árvores com DAP maior que $25 \mathrm{~cm}$ foram numeradas seqüencialmente em cadá sub-bloco e etiquetadas com placas de alumínio.

Para efeito de simulação, foram tomadas 40 unidades amostrais de cada tamanho proposto. A tomada das unidades foi feita através da probabilidade de seleção igual para todas as parcelas, aleatoriamente. Optou-se pela tomada aleatória porque, com as modernas técnicas de interpretação de fotografias aéreas e imagens de satélite, dificilmente se utilizará a amostragem não aleatória, onde as parcelas não são escolhidas pelas leis da probabilidade mas pelo julgamento pessoal ou sistematicamente.

Os tamanhos propostos são de larguras de $12,5 \mathrm{~m}$. em múltiplos de 12,5 , até $50 \mathrm{~m}$ e comprimentos de $25 \mathrm{~m}$, em múltiplos de 25 , até $200 \mathrm{~m}$.

A escolha do melhor tamanho proposto foi feita baseada na consideração do custo de medição e precisão desejada, através da Eficiência Relativa (ER), proposta por Husch $\epsilon t$ al. (1971).

$$
E R=\frac{\text { (se) } 1 . C_{1}}{\text { (se) } 2 . C_{2}} \cdot 100
$$

Para reforçar a comparação entre diferentes tamanhos de parcelas amostrais, foram ainda calculados o Limite de Erro (LE), o Coeficiente de Variação (CV), o número de parcelas $(n)$ para o Inventário definitivo e a área total de amostragem (An).

$$
\begin{aligned}
L E & =\frac{s \bar{x} \cdot t}{\bar{x}} \\
C V & =\frac{s}{\bar{x}} \cdot 100
\end{aligned}
$$




$$
\begin{aligned}
& n=\frac{N \cdot t^{2} \cdot(C V)^{2}}{N(L E \%)+t^{2}(C V)^{2}} \begin{array}{l}
\text { para populações } \\
\text { finitas }
\end{array} \\
& n=\frac{t^{2}(C V)^{2}}{(L E \%)^{2}} \quad \begin{array}{l}
\text { para populações in- } \\
\text { finitas }
\end{array}
\end{aligned}
$$

Onde :

(se) 1: desvio padrão de um tamanho de parcela tomado como base de comparação, neste caso, a parcela de $25 \times 200 \mathrm{~m}$.

(se) 2: desvio padrão de um tamanho de parcela que está sendo comparada.

C1: custo de medição na parcela básica

C2: custo de medição na parcela que está sendo comparada

$s \bar{x}$ : erro padrão da média

$t$ : valor da tabela de Student a $95 \%$ de probabilidade

s: estimativa do desvio padrão

$\mathrm{N}$ : número de ınidades potenciais que a população pode ser dividida

$\mathrm{n}$ : número de amostras para o Inventário Florestal definitivo

custo de medição $=$ custo fixo + custo variável inerente a cada parcela (neste trabalho foram considerados, custo fixo $=\mathrm{Cr} \$ 6.000,00$ por parcela e custo variável $=\operatorname{Cr} \$ 12,50$ por minuto).

Populações infinitas: $f=1-\frac{n}{N}$ maior ou igual a 0,98

Populações finitas: $f$ menor que 0,98

O erro padrão calculado no presente trabaIho e todas as outras estimativas se referem à área basal em $\mathrm{m}^{2}$ de cada parcela. Foi utilizada a área basal por unidade de área como referência porque, segundo Husch et al. (1971), é uma característica muito útil de um povoamento florestal, estando diretamente relacionada ao volume e é também uma medida de densidade do povoamento. Segundo ainda o mesmo autor, densidade do povoamento é uma meáida quantitativa de um maciço florestal em termos de área basal, refletindo o grau de ocupação das árvores dentro de uma determinacia área.

Foi também avaliado o relacionamento entre o Coeficiente de Variação $\in \sigma$ tamanho de parcela amostral, através da equação de re. gressão do tipo $Y=a+b(1 / X)$, na tentativa de comprovar a diminuição do CV com o aumento do tamanho da parcela amostral e diferentes distribuições espaciais. As equações para a determinação dos coeficientes de regressão, equações normais, foram obtidas através do método dos mínimos quadrados.

Todo o estudo foi realizado, separadamente, para o total (TOT) de espécies florestais que ocorrem na área, as espécies listadas (EL) e para as outras (OUT). A lista de espécies listadias foi feita, após consultas a várias serrarias de Manaus e é apresentada na Tabela 3.

\section{Resultados E DISCUSSõeS}

Do Inventáric Florestal a $100 \%$ da área em estudo, os seguintes parâmetros (área basal por hectare) foram determinados :

\begin{tabular}{lrrrc} 
& \multicolumn{1}{c}{$\mu$} & \multicolumn{1}{c}{$\sigma^{2}$} & $\sigma$ & $\mathrm{CV}(\%)$ \\
TOT & 18,32 & 15,26 & 3,90 & 21 \\
EL & 5,99 & 2,21 & 1,49 & 25 \\
OUT & 12,33 & 10,94 & 3,31 & 27
\end{tabular}

Esses valores representam o potencial médio de uma área de 80 hectares considerada, para efeito de simulaçõs, como população folrestal de árvores com DAP maior ou igual a $25 \mathrm{~cm}$. Transformando a média do total (TOT), de área basal em volume cúbico por hectare, tem-so aproximadamente $175 \mathrm{~m}^{3} /$ ha. Esse valor médio está entre as médias obtidas em outras partes da floresta amazônica, por exemplo: Rodovia Belém-Brasília, trecho Imperatriz a Belém $\left(151 \mathrm{~m}^{3} / \mathrm{ha}\right.$ - DAP maior que $\left.25 \mathrm{~cm}\right)$, Curuá-Una $\left(164 \mathrm{~m}^{3} / \mathrm{ha}\right.$ - DAP maior que $\left.45 \mathrm{~cm}\right)$, Floresta entre os rios Tapajós e Xingu $\left(210 \mathrm{~m}^{3}\right.$ / ha - DAP maior que $25 \mathrm{~cm}$ ), Projeto Integrado de Colonização de Altamira $\left(146 \mathrm{~m}^{3} / \mathrm{ha}\right.$ - DAP maior que $20 \mathrm{~cm}$ ) e na área do Projeto Jari $\left(300 \mathrm{~m}^{3} / \mathrm{ha}\right.$ - DAP maior que $\left.20 \mathrm{~cm}\right)$. 
TABELA 3 - Espécies Listadas (EL)

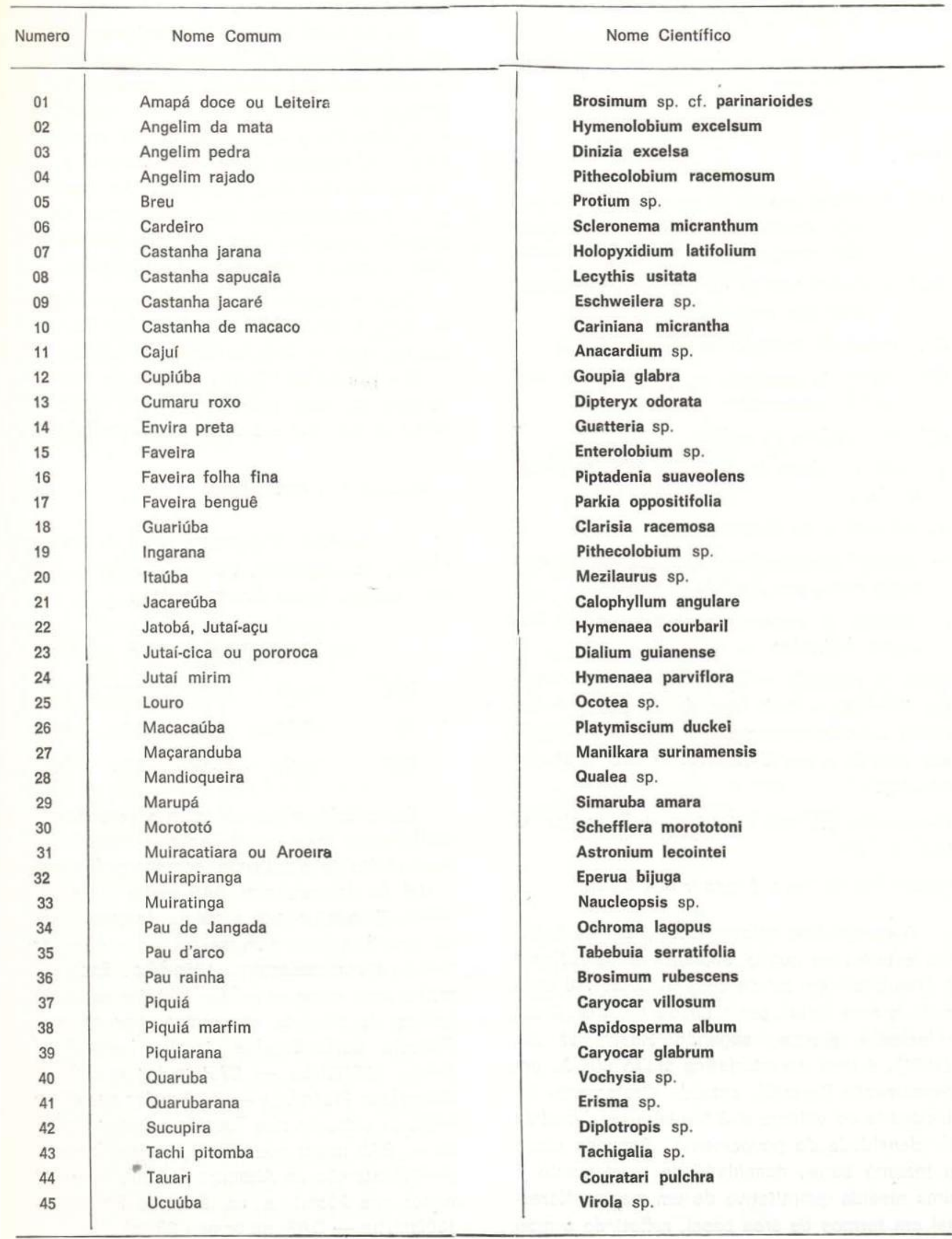


As estimativas de média e variância, obtidas des simulações em 40 parcelas amostrais sãc apresentadas na Tabela 4, com os devidos tamanhos para TOT, EL e OUT.

Nas Tabelas 5 e 5 a, são apresentados os valores calculados, a partir dos estimados da Tabela 4, de Coeficiente de Variação (CV). Eficiência Relativa (ER), Limite de Erro (LE), número de amostras $(n)$ e área total de amostragem para cada tamanho de parcela (An). Cada tabela representa a simulação de diferentes tamanhos de parcela amostral de larguras e comprimentos variados, separadamente para TOT, EL e OUT.

A montagem da Tabela 6 foi feita através de eliminações, sendo que, na primeira foi considerado o Limite de Erro menor ou igual a $10 \%$ nas 3 categorias, TOT, EL e OUT.

Dos 32 diferentes tamanhos de parcelas amostrais testados, somente 13 atenderam a primeira condicionante (LE menor ou igual a $10 \%)$, destacando a parcela $n^{\circ} 32(50 \times 200 m)$ com 4, 6 e 5 de LE, respectivamente para TOT, EL e OUT e, $\mathrm{Gm}$ seguida, aparece a parcela no $22(37,5 \times 150 m)$ com 5,5 e 6 , o que representa uma diferença mínima para LE entre as duas parcelas.

Os CV estimados para os 13 tamanhos listados na Tabela 6, segunda condicionante, são relativamente baixos em se tratando de floresta amazônica (CV em torno de $40 \%$ ), podendo destacar a parcela $n^{\circ} 22$, a qual apresentou o CV menor que o da população, nas 3 categorias.

Em termos de ER sobre a parcela base $(25 \times 200 \mathrm{~m})$, somente 3 parcelas apresentaram ER maior que $100 \%$ nas 3 categorias juntas, sendo, por esta razão, mais eficientes que a parcela base ( $n^{0}$ 16) e são: $n .^{0} 21(37,5 \times 125 m)$, n. ${ }^{0} 22$ e n. $^{\circ} 28(50 \times 100 m)$.

Quanto à área total de amostragem necessária para atender um LE pré-fixado de $10 \%$, destaca novamente a parcela $\mathrm{n}^{0} 22$ com $7,38-9,80-12,45$ ha, respectivamente para TOT, EL e OUT, seguida das parcelas $n^{\circ} 21 \mathrm{com}$ $8,90-12,03-14,11, n^{\circ} 27(50 \times 75 \mathrm{~m})$ com 8,49-12,7313,15 e a $n^{\circ} 20(37,5 \times 100 m)$ com $8,84-15,40$ 12,23ha.
Na tigura 1 é apresentado o relacionamento entre o CV e os diferentes tamanhos de parcelas amostrais, a partir da equação da hipérbole. Nas 3 categorias, TOT, EL e OUT, podem ser observados que os CV diminusm com o aumento do tamanho das parcelas. Na categoria TOT, o CV ganha certa estabilidade a partir de parceias com $2000 \mathrm{~m}^{2}$, estando entre 20 a $30 \%$. Em EL, a partir de $3000 \mathrm{~m}^{2} \mathrm{com}$ CV entre 30 a $40 \%$ e em OUT, a partir de $2500 \mathrm{~m}^{2}$, com CV aproximadamente de $30 \%$.

A respeito de forma da parcela, observa-se que na Tabela 9, o menor comprimento apresentado é de $75 \mathrm{~m}$ para a maior largura $(50 \mathrm{~m})$. Considerando as 13 parcelas juntas, tem-se um comprimento médio de $150 \mathrm{~m}$.

\section{CONCLUSÃo}

Matematicamente, as dimensões ideais de parcela, para o Inventário Florestal da população florestal em estudo, são $37,5 \mathrm{~m}$ por $150 \mathrm{~m}$ porque, em todos os fatores tomados como comparadores, foi esta parcela que melhor se apresentou nas 3 categorias, TOT, EL e OUT, com menores CV, LE \& área amostral e, maior ER em relação à parcela base $(25 \times 200 \mathrm{~m})$.

Entretanto, em se tratando de Inventário Florestal, de uma maneira geral, outros fatcres também devem ser levados em consideração, além da Matemática. Na figura 1, pode ser observado que, a partir de parcelas de $3000 \mathrm{~m}^{2}$. existe pouco ganho de precisão do trabalho, englobando as 3 categorias, TOT, EL e OUT. Com o CV estabilizando-se, o erro amostral também tende a estabilizar-se mas, os erros não amostrais terão, nesse caso, uma tendência contrária. Naturalmente, quanto maior for a parcela amostral, maior será a tendência de cometerem-se erros não amostrais face às dificuldades na supervisão dos trabalhos de campo. Do ponto de vista econômico, os custos diminuem com a diminuição do tamanho das parcelas, principalmente os custos de medição. E mais: na maioria dos Inventários Florestais, os LE de $10 \%$, alguns com até $20 \%$, produzem resultados confiáveis. 
TABELA 4 - Estimativas obtidas no Inventário florestal de 40 parcelas amostrais

\begin{tabular}{|c|c|c|c|c|c|c|c|c|}
\hline \multirow{2}{*}{$P$} & \multirow{2}{*}{$\begin{array}{l}\text { DIMENSŌES } \\
(m \times m)\end{array}$} & \multirow{2}{*}{ AREA $\left(\mathrm{m}^{2}\right)$} & \multicolumn{2}{|c|}{ TOT } & \multicolumn{2}{|c|}{ EL } & \multicolumn{2}{|c|}{ OUT } \\
\hline & & & $\bar{x}$ & $s^{2}$ & $\bar{x}$ & $\mathrm{~s}^{2}$ & $\bar{x}$ & $\mathrm{~s}^{2}$ \\
\hline 1 & $12,5 \times 25$ & 312,5 & 0,43 & 0,06 & 0,14 & 0,02 & 0,29 & 0,05 \\
\hline 2 & $12,5 \times 50$ & 625 & 1,23 & 0,35 & 0,38 & 0,11 & 0,85 & 0,19 \\
\hline 3 & $12,5 \times 75$ & 937,5 & 1,65 & 0,37 & 0,52 & 0,09 & 1,13 & 0,25 \\
\hline 4 & $12,5 \times 100$ & 1250 & 2,27 & 0,63 & 0,71 & 0,25 & 1,55 & 0,36 \\
\hline 5 & $12,5 \times 125$ & 1562,5 & 2,72 & 0,57 & 0,87 & 0,19 & 1,84 & 0,56 \\
\hline 6 & $12,5 \times 150$ & 1875 & 3,59 & 1,32 & 1,14 & 0,40 & 2,45 & 0,73 \\
\hline 7 & $12,5 \times 175$ & 2187,5 & 4,19 & 1,69 & 1,41 & 0,48 & 2,75 & 0,95 \\
\hline 8 & $12,5 \times 200$ & 2500 & 4,36 & 1,16 & 1,40 & 0,36 & 2,97 & 0,92 \\
\hline 9 & $25 \times 25$ & 625 & 1,24 & 0,26 & 0,38 & 0,10 & 0,87 & 0,19 \\
\hline 10 & $25 \times 50$ & 1250 & 2,29 & 0,62 & 0,72 & 0,19 & 1,57 & 0,38 \\
\hline 11 & $25 \times 75$ & 1875 & 3,52 & 1,26 & 1,12 & 0,42 & 2,40 & 0,88 \\
\hline 12 & $25 \times 100$ & 2500 & 4,67 & 1,58 & 1,64 & 0,65 & 3,03 & 1,06 \\
\hline 13 & $25 \times 125$ & 3125 & 5,74 & 2,83 & 1,87 & 0,44 & 3,87 & 1,96 \\
\hline 14 & $25 \times 150$ & 3750 & 7,11 & 2,82 & 2,43 & 0,88 & 4,68 & 1,63 \\
\hline 15 & $25 \times 175$ & 4375 & 7,99 & 3,21 & 2,51 & 0,78 & 5,48 & 2,33 \\
\hline 16 & $25 \times 200$ & 5000 & 9,40 & 5,31 & 2,99 & 0,75 & 6,42 & 4,70 \\
\hline 17 & $37,5 \times 25$ & 937,5 & 1,84 & 0,32 & 0,61 & 0,14 & 1,23 & 0,19 \\
\hline 18 & $37,5 \times 50$ & 1875 & 3,42 & 1,62 & 1,12 & 0,40 & 2,30 & 0,96 \\
\hline 19 & $37,5 \times 75$ & 2812,5 & 5,15 & 1,95 & 1,63 & 0,57 & 3,52 & 1,16 \\
\hline 20 & $37,5 \times 100$ & 3750 & 7,15 & 3,33 & 2,36 & 0,71 & 4,79 & 2,19 \\
\hline 21 & $37,5 \times 125$ & 4687,5 & 8,26 & 3,94 & 2,75 & 0,64 & 5,51 & 3,19 \\
\hline 22 & $37,5 \times 150$ & 5625 & 10,50 & 4,09 & 3,34 & 0,58 & 7,16 & 3,57 \\
\hline 23 & $37,5 \times 175$ & 6562,5 & 11,55 & 7,49 & 3,63 & 0,98 & 7,92 & 5,86 \\
\hline 24 & $37,5 \times 200$ & 7500 & 13,61 & 11,38 & 4,45 & 1,20 & 9,19 & 7,41 \\
\hline 25 & $50 \times 25$ & 1250 & 2,25 & 0,57 & 0,75 & 0,16 & 1,50 & 0,51 \\
\hline 26 & $50 \times 50$ & 2500 & 4,52 & 1,54 & 1,45 & 0,39 & 3,07 & 1,08 \\
\hline 27 & $50 \times 75$ & 3750 & 6,37 & 2,62 & 2,00 & 0,42 & 4,37 & 2,10 \\
\hline 28 & $50 \times 100$ & 5000 & 9,11 & 4,21 & 3,06 & 0,64 & 6,05 & 3,50 \\
\hline 29 & $50 \times 125$ & 6250 & 11,52 & 6,25 & 3,71 & 1,18 & 7,81 & 5,30 \\
\hline 30 & $50 \times 150$ & 7500 & 13,52 & 10,79 & 4,38 & 1,65 & 9,14 & 7,68 \\
\hline 31 & $50 \times 175$ & 8750 & 16,00 & 11,60 & 5,11 & 2,39 & 10,89 & 8,67 \\
\hline 32 & $50 \times 200$ & 10000 & 19,00 & 12,13 & 5,77 & 2,33 & 13,23 & 9,61 \\
\hline
\end{tabular}


TABELA 5 - Resumo dos cálculos de CV, ER, LE, An e n para parcelas com larguras de 12,5 e $25 \mathrm{~m}$ e comprimentos variados de $25 \mathrm{~m}$, em múltiplos de 25 , até $200 \mathrm{~m}$

\begin{tabular}{|c|c|c|c|c|c|c|c|c|c|c|c|c|c|c|c|c|}
\hline & & \multicolumn{15}{|c|}{12,5} \\
\hline & & & & TOT & & & & & EL & & & & & OUT & & \\
\hline & $\mathrm{N} .^{\circ}$ & CV & ER & LE & $n$ & An & CV & ER & LE & $n$ & An & CV & ER & LE & $n$ & $\mathrm{An}$ \\
\hline 25 & 1 & 55,81 & 49,59 & 0,19 & 127,08 & 3,97 & 100,00 & 32,91 & 0,29 & 408,00 & 12,75 & 75,86 & 50,39 & 0,21 & 234,79 & 7,34 \\
\hline 50 & 2 & 47,97 & 57,20 & 0,15 & 93,89 & 5,87 & 89,47 & 36,47 & 0,27 & 326,60 & 20,41 & 51,76 & 73,22 & 0,17 & 109,31 & 6,83 \\
\hline 75 & 3 & 36,97 & 73,58 & 0,11 & 51,30 & 4,81 & 57,69 & 56,07 & 0,19 & 112,02 & 10,50 & 44,25 & 84,91 & 0,14 & 71,02 & 6,66 \\
\hline 100 & 4 & 34,80 & 77,50 & 0,11 & 45,87 & 5,73 & 70,42 & 45,54 & 0,23 & 153,73 & 19,22 & 38,71 & 96,24 & 0,12 & 55,81 & 6,98 \\
\hline 125 & 5 & 27,94 & 95,71 & 0,08 & 28,97 & 4,53 & 49,43 & 64,34 & 0,14 & 76,01 & 11,88 & 40,76 & 90,62 & 0,12 & 55,94 & 8,74 \\
\hline 150 & 6 & 32,03 & 82,79 & 0,10 & 37,02 & 6,94 & 55,26 & 57,07 & 0,16 & 89,68 & 16,82 & 35,10 & 104,36 & 0,11 & 43,44 & 8,15 \\
\hline 175 & 7 & 31,03 & 84,75 & 0,09 & 34,99 & 7,65 & 48,94 & 63,90 & 0,14 & 74,86 & 16,38 & 35,64 & 101,92 & 0,11 & 44,60 & 9,76 \\
\hline 200 & 8 & 24,77 & 105,29 & 0,07 & 23,22 & 5,81 & 42,86 & 72,37 & 0,13 & 60,73 & 15,18 & 32,32 & 111,47 & 0,10 & 37,61 & 9,40 \\
\hline
\end{tabular}

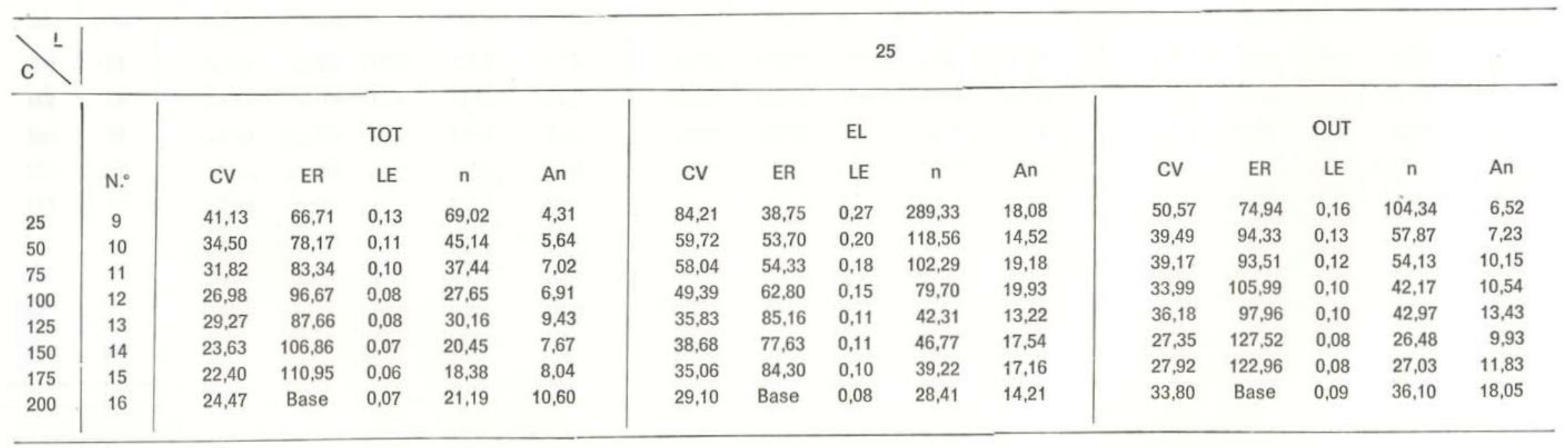

\footnotetext{
L: Largura da parcela
}

| C: Comprimento da parcela 

até $200 \mathrm{~m}$

\begin{tabular}{|c|c|c|c|c|c|c|c|c|c|c|c|c|c|c|c|c|}
\hline$L$ & & \multicolumn{15}{|c|}{37,5} \\
\hline & \multicolumn{16}{|c|}{ OUT } \\
\hline & $\mathrm{N} .^{\circ}$ & CV & ER & LE & $\mathrm{n}$ & An & CV & ER & LE & $n$ & An & CV & ER & LE & $n$ & An \\
\hline 25 & 17 & 30,98 & 87,80 & 0,10 & 37,33 & 3,50 & 60,66 & 53,33 & 0,20 & 126,41 & 11,85 & 35,77 & 105,04 & 0,12 & 49,01 & 4,59 \\
\hline 50 & 18 & 37,13 & 71,42 & 0,11 & 49,31 & 9,25 & 57,14 & 55,19 & 0,18 & 99,93 & 18,74 & 42,61 & 85,96 & 0,13 & 62,50 & 11,72 \\
\hline 75 & 19 & 27,18 & 95,18 & 0,08 & 26,19 & 7,37 & 46,63 & 65,97 & 0,14 & 61,45 & 17,28 & 30,68 & 116,47 & 0,09 & 32,22 & 9,06 \\
\hline 100 & 20 & 25,59 & 98,67 & 0,07 & 23,57 & 8,84 & 35,59 & 85,37 & 0,10 & 41,07 & 15,40 & 30,90 & 112,87 & 0,09 & 32,61 & 12,23 \\
\hline 125 & 21 & 23,97 & 102,88 & 0,06 & 18,99 & 8,90 & 29,09 & 100,81 & 0,07 & 25,66 & 12,03 & 32,49 & 104,84 & 0,08 & 30,10 & 13,11 \\
\hline 150 & 22 & 19,24 & 125,25 & 0,05 & 13,12 & 7,38 & 22,75 & 125,97 & 0,05 & 17,43 & 9,80 & 26,40 & 126,08 & 0,06 & 22,14 & 12,45 \\
\hline 175 & 23 & 23,72 & 99,33 & 0,06 & 18,67 & 12,25 & 27,27 & 102,74 & 0,07 & 23,28 & 15,28 & 30,56 & 106,49 & 0,07 & 27,59 & 18,11 \\
\hline 200 & 24 & 24,72 & 93,23 & 0,06 & 19,96 & 14,97 & 24,72 & 110,87 & 0,06 & 19,96 & 14,97 & 29,60 & 107,54 & 0,07 & 26,33 & 19,75 \\
\hline
\end{tabular}

\begin{tabular}{|c|c|c|c|c|c|c|c|c|c|c|c|c|c|c|c|c|}
\hline$\checkmark L$ & & & & & & & \multicolumn{5}{|c|}{50} & & & & & \\
\hline & & \multicolumn{5}{|c|}{ TOT } & \multicolumn{5}{|c|}{ EL } & \multicolumn{5}{|c|}{ OUT } \\
\hline & $\mathrm{N} .^{\circ}$ & CV & ER & LE & $n$ & An & CV & ER & LE & $n$ & An & $\mathrm{CV}$ & ER & LE & $n$ & An \\
\hline 25 & 25 & 33,78 & 78,84 & 0,11 & 43,40 & 5,43 & 53,33 & 60,14 & 0,16 & 98,23 & 12,28 & 47,33 & 78,71 & 0,15 & 79,98 & 10,00 \\
\hline 50 & 26 & 27,43 & 95,08 & 0,08 & 28,01 & 7,00 & 42,76 & 72,54 & 0,13 & 60,50 & 15,13 & 33,88 & 106,33 & 0,10 & 40,85 & 10,21 \\
\hline 75 & 27 & 25,43 & 99,29 & 0,07 & 22,65 & 8,49 & 32,50 & 92,39 & 0,09 & 33,95 & 12,73 & 33,18 & 105,12 & 0,09 & 35,07 & 13,15 \\
\hline 100 & 28 & 22,50 & 108,76 & 0,06 & 18,29 & 9,15 & 26,14 & 111,32 & 0,07 & 23,74 & 11,87 & 30,91 & 109,35 & 0,09 & 31,34 & 15,67 \\
\hline 125 & 29 & 21,70 & 109,39 & 0,05 & 16,12 & 10,08 & 29,38 & 96,08 & 0,07 & 26,05 & 16,28 & 29,45 & 111,33 & 0,07 & 26,14 & 16,34 \\
\hline 150 & 30 & 24,26 & 95,00 & 0,06 & 19,36 & 14,52 & 29,22 & 93,79 & 0,07 & 25,84 & 19,38 & 30,31 & 105,03 & 0,08 & 27,26 & 20,45 \\
\hline 175 & 31 & 21,31 & 105,09 & 0,05 & 15,04 & 13,16 & 30,33 & 87,81 & 0,07 & 25,55 & 22,36 & 27,00 & 114,57 & 0,06 & 21,68 & 18,97 \\
\hline 200 & 32 & 18,32 & 118,88 & 0,04 & 11,69 & 11,69 & 26,34 & 98,33 & 0,06 & 20,91 & 20,91 & 23,43 & 128,39 & 0,05 & 17,50 & 17,50 \\
\hline
\end{tabular}


TABELA 6 - Parcelas que apresentaram LE menor ou igual a $10 \%$ em TOT, EL e OUT.

\begin{tabular}{|c|c|c|c|c|c|c|c|c|c|c|c|c|c|c|c|}
\hline$P$ & & LE & & & CV & & & ER & & & $n$ & & & An & \\
\hline$P$ & TOT & $\mathrm{EL}$ & OUT & TOT & EL & OUT & TOT & EL & OUT & TOT & EL & OUT & TOT & EL & OUT \\
\hline 15 & 6 & 10 & 8 & 22,40 & 35,06 & 27,92 & 110,95 & 84,30 & 122,96 & 18,38 & 39,22 & 27,03 & 8,04 & 17,16 & 11,83 \\
\hline 16 & 7 & 8 & 9 & 24,47 & 29,10 & 33,80 & Base & Base & Base & 21,19 & 28,41 & 36,10 & 10,60 & 14,21 & 18,05 \\
\hline 21 & 6 & 7 & 8 & 23,97 & 29,09 & 32,499 & 102,88 & 100,81 & 104,84 & 18,99 & 25,66 & 30,10 & 8,90 & 12,03 & 14,11 \\
\hline 22 & 5 & 5 & 6 & 19,24 & 22,75 & 26,40 & 125,25 & 125,97 & 126,08 & 13,12 & 17,43 & 22,14 & 7,38 & 9,80 & 12,45 \\
\hline 23 & 6 & 7 & 7 & 23,72 & 27,27 & 30,56 & 99,33 & 102,74 & 106,49 & 18,67 & 23,28 & 27,59 & 12,25 & 15,28 & 18,11 \\
\hline 24 & 6 & 6 & 7 & 24,72 & 24,72 & 29,60 & 93,23 & 110,87 & 107,54 & 19,96 & 19,96 & 26,33 & 14,97 & 14,97 & 19,75 \\
\hline 27 & 7 & 9 & 9 & 25,43 & 32,50 & 33,18 & 99,29 & 92,39 & 105,12 & 22,65 & 33,95 & 35,07 & 8,49 & 12,73 & 13,15 \\
\hline 28 & 6 & 7 & 9 & 22,50 & 26,14 & 30,91 & 108,76 & 111,32 & 109,35 & 18,29 & 23,74 & 31,34 & 9,15 & 11,87 & 15,67 \\
\hline 29 & 5 & 7 & 7 & 21,70 & 29,38 & 29,45 & 109,39 & 96,08 & 111,33 & 16,12 & 26,05 & 26,14 & 10,08 & 16,28 & 16,34 \\
\hline 30 & 6 & 7 & 8 & 24,26 & 29,22 & 30,31 & 95,00 & 93,79 & 105,03 & 19,36 & 25,84 & 27,26 & 14,52 & 19,38 & 20,45 \\
\hline 31 & 5 & 7 & 6 & 21,31 & 30,33 & 27,00 & 105,09 & 87,81 & 114,57 & 15,04 & 25,55 & 21,68 & 13,16 & 22,36 & 18,97 \\
\hline 32 & 4 & 6 & 5 & 18,32 & 26,34 & 23,43 & 118,88 & 98,33 & 128,39 & 11,69 & 20,91 & 17,50 & 11,69 & 20,91 & 17,50 \\
\hline
\end{tabular}




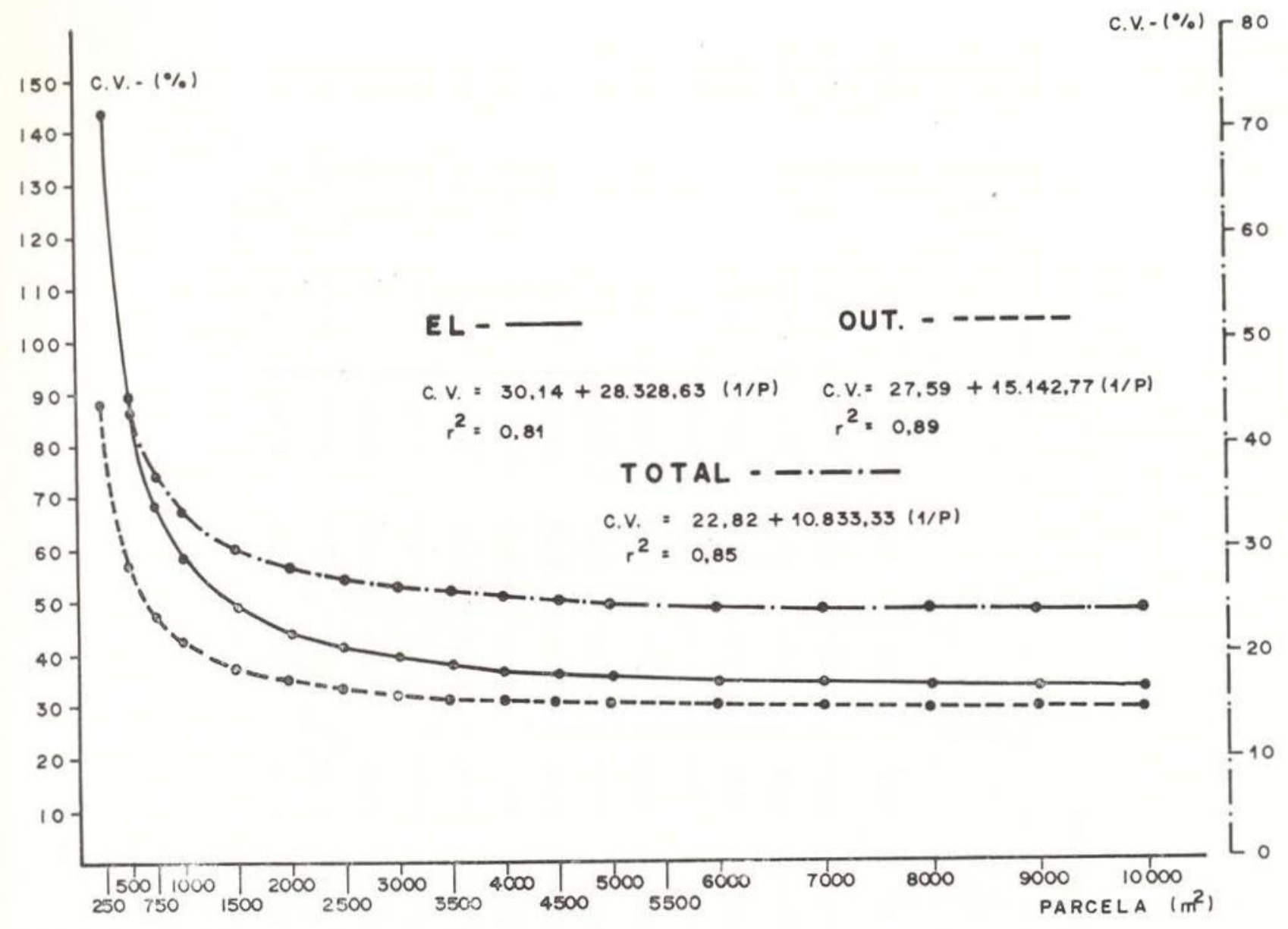

Fig. 1 - Relacionamento entre Coeficiente de Variação e tamanho de parcelas amostrais.

Sendo assim, pode concluir-se que, para a populaçäo florestal em estudo, 80 hectares e somente com árvores de DAP maior ou igual a $25 \mathrm{~cm}$, a melhor parcela é a $\mathrm{n}^{0} 22(37,5 \times 150 \mathrm{~m})$. Para efeito de Inventários Florestais do Distrito Agropecuário da SUFRAMA, entretanto qualquer uma das parcelas da Tabela 6 pode ser usada, de acordo com a conveniência. E, para um Inventário Florestal mais abrangente do que o do Distrito, a tendência das curvas (Relacionamento CV e tamanho de parcela), figura 1 , pode ser usada, a partir de $3000 \mathrm{~m}^{2}$ para as 3 categorias, TOT, EL e OUT.

Quanto à forma de parcela, a retangular se apresentou melhor que a quadrada, podendo ser utilizada, de uma maneira geral, parcela de largura de atẻ $37,5 \mathrm{~m}$ para um comprimento de 150 metros, nos futuros trabalhos de Inventáric Florestal no Distrito Agropecuário.

\section{SUMMARY}

This work is the result of an investigation concerning the best size for a Forest Inventory sample plot in the Manaus region. This plot was developed from 32 different size plots simulated one by one on an 80 hectare forest area, after complete enumeration of those trees with more than $25 \mathrm{~cm}$ DBH. In this case the best plot was number 22 with dimensions at $37.5 \mathrm{~m}$ by 150 metres.

\section{REFERENCIAS BIBLIOGRAFICAS}

BERRY, M.J. \& HOWARD, W.J.

1973 - Fiji Forest Inventory. Land Resource Study n. 12. Subirton, Surrey, England. 98p.

DE MILDE, R.A.J.

1975 - Surinam Forest Inventory. FO:DP/SUR/71/ 506 Technical Report 6. FAO of the United Nations, Paramaribo.

FAO

1974 - Manual de Inventárlo Florestal (con especial referência a los bosques mixtos tropicals). Roma. 195p. 
FREESE, $F$.

1967 - Elomentary statistical mothods for fores. ters. Washington. Forest Service. 87p.

GLERUM, B.B. \& SMIT, G.

1965a - Inventário Florestal total na região do CuruáUna. Inventários Florestais na Amazônia. Rio de Janeiro, SPEVEA, v. 7, 51p.

1965b_ Pesquisa combinada Floresta-Solo no Pará e Maranhão. Inventários Florestais na Amazônia. Rio de Janeiro, SPEVEA, v. 9, 113p.

HEINSDIJK, D.

1965 - A Floresta entre os rios Tapajós e Xingu. Inventários Florestais na Amazônia. Rio de Janeiro, SPEVEA, v. 1, 68p.

HUSCH, B.; MILLER, C.I. \& BEERS, T.W.

1971 - Forest mensuration. New York, Ronald Press, $410 p$

LOETSCH, F.; ZÖHRER, F. \& HALLER, K.E.

1973 - Forest Inventory. München, BLV Verlagsgeselischaft, Wien, v. 2.
QUEIROZ, W.T.

1977 - Efeitos da variação estrutural em unidade amostral na aplicação do processo de amostragem em Conglomerados nas florestas do Planalto do Tapajós. Revista Floresta, 8 (1): 19-23.

U.F.Pr. Centro de Pesquisas Florestais

1972 - Inventário Florestal do Distrito Federal. Curitiba, 158p.

1976 - Inventário Floresta do Projeto Integrado de Colonização de Altamira. Curitiba, 127p.

1978a - Inventário Florestal da região da influência da represa de Itaipu. Curitiba, 177p.

1978b- Inventário Florestal do Pinheiro no Sul do Brasil. Curitiba, 327p.

1980 - Determinação da produção volumétrica dos plantios de Eucalyptos e Pinus nos Estados de Minas Gerais, Goiás e Mato Grosso do Sul. Curitiba, 203p.

(Aceito para publicação em $02 / 10 / 81$ ) 